Journal of Advanced Research in Fluid Mechanics and Thermal Sciences

\title{
Meshless Fluid Structural Interaction (FSI) Simulation of Deformation of Flexible Structure due to Water Dam Break
}

\author{
Khai Ching $\mathrm{Ng}^{1,}{ }^{*}$, Lit Ken $\mathrm{Tan}^{2}$, Wah Yen $\mathrm{Tey}^{2,3}$ \\ Department of Mechanical, Materials and Manufacturing Engineering, The University of Nottingham Malaysia Campus, Jalan Broga, 43500 \\ Semenyih, Selangor Darul Ehsan, Malaysia \\ 2 Takasago i-Kohza, Malaysia-Japan International Institute of Technology, Universiti Teknologi Malaysia, Jalan Sultan Yahya Petra, Kampung Datuk \\ Keramat, 54100 Kuala Lumpur, Malaysia \\ 3 Department of Mechanical Engineering, 1, Jalan Puncak Menara Gading, Taman Connaught, 56000 Kuala Lumpur, Malaysia
}

\section{ARTICLE INFO}

\section{Article history:}

Received 16 December 2019

Received in revised form 20 March 2020

Accepted 20 March 2020

Available online 30 April 2020

\section{Keywords:}

Meshless; Smoothed Particle

Hydrodynamics (SPH); Lattice-Spring

Model (LSM); Fluid Structural Interaction

(FSI); particle method

\section{ABSTRACT}

A new meshless-based solver has been used to study the deformation of flexible structure due to water dam break. This meshless Fluid Structural Interaction (FSI) solver couples the Smoothed Particle Hydrodynamics (SPH) and Lattice-Spring Model (LSM). SPH and LSM are used to model the motions of fluid and solid particles, respectively. As both are essentially particle-based methods, the force coupling at the interface is straightforward. The numerical results have been compared with the benchmark numerical solutions of dam break problem and good agreement has been found.

\section{Introduction}

The numerical method for simulating fluid flow can be broadly classified into two categories. The first category refers to those methods that require discretization of the entire flow domain using polygons (or meshes), or more-specifically known as the mesh-based methods. One of the common mesh-based methods is the Finite Volume Method [1-12], which is very popular amongst the CFD users. The second category does not require the use of polygons (mesh); however, computation is performed using zero-dimensional points (mesh-less method). Some examples are Moving Particle Semi-implicit (MPS) method [13-15], hybrid particle-mesh methods [16-19], Dissipative Particle Dynamics (DPD) [20, 21] and Smoothed Particle Hydrodynamics (SPH) [22-24], to name a few. SPH is one of the oldest meshless methods that has been gaining popularity in CFD nowadays.

\footnotetext{
${ }^{*}$ Corresponding author.

E-mail address: ngkhaiching2000@yahoo.com (Khai-Ching Ng)
} 
Likewise, the methods available in simulating solid mechanics can be broadly classified into meshbased and mesh-less methods. Undoubtedly, Finite Element Method (FEM) can be regarded as the popular mesh-based method in studying solid deformation. Recently, the use of meshless method such as Lattice Spring Model (LSM) has been gaining popularity as well [25]. The accuracy of LSM has been proven to be at par with that of FEM $[26,27]$.

In the current work, we intend to use the coupled SPH-LSM meshless method in simulating Fluid Structure Interaction (FSI) problem. Specifically, we intend to study the accuracy of SPH-LSM method in solving the dam-break flow involving a flexible gate.

\section{Methodology}

In the current work, the continuity and momentum equations are discretized using SPH:

$\frac{d \rho_{i}}{d t}=\rho_{i} \sum_{j} V_{j}\left(\mathbf{v}_{i}-\mathbf{v}_{j}\right) \cdot \nabla_{i} W_{i j}$

$m_{i} \frac{d \mathbf{v}}{d t}=-\sum_{j}\left(V_{i}^{2}+V_{j}^{2}\right) \frac{P_{i} \rho_{j}+P_{j} \rho_{i}}{\rho_{i}+\rho_{j}} \nabla_{i} W_{i j}+\sum_{j}\left(V_{i}^{2}+V_{j}^{2}\right) \frac{2 \mu_{i} \mu_{j}}{\mu_{i}+\mu_{j}} \frac{\mathbf{v}_{i}-\mathbf{v}_{j}}{\left\|\mathbf{r}_{i j}\right\|} \nabla_{i} W_{i j} \cdot \frac{\mathbf{r}_{i j}}{\left\|\mathbf{r}_{i j}\right\|}+m_{i} \mathbf{g}$

where $V$ is the fluid volume, $m$ is the fluid mass, $\mathbf{v}$ is the fluid velocity vector, $\rho$ is the fluid density, $P$ is the fluid pressure, $\boldsymbol{r}_{i j}$ denotes $\boldsymbol{r}_{i}-\boldsymbol{r}_{\mathbf{j}}, \boldsymbol{g}$ is the gravitational vector and $\mu$ is the fluid viscosity. The quintic spline kernel function [28] was used to represent $W$.

In order to model the solid motion, each solid particle is connected with its neighbouring solid particles via springs as shown in Figure 1. Here, the spring stiffness for horizontal and vertical springs are denoted as $k_{L}$ and the spring stiffness for diagonal spring is denoted as $k_{D}$. Following the LSM method [26] for 2D plane strain problem, the following spring stiffness values can be obtained:

$k_{L}=\frac{2 E}{1+v}$

$k_{D}=\frac{E}{1+v}$

$T=\frac{E(4 v-1)}{(1+v)(1-2 v)(2+\sqrt{2})^{2}}$.

Here, the stiffness $T$ is introduced to simulate the elastic behaviour at arbitrary Poisson ratio. These stiffness values are dependent on Young's modulus $E$ and Poisson ratio $v$. The elastic force acting on the solid particle $I$ (see Figure 1 ) can then be computed as:

$\mathbf{F}_{S, I}=\sum_{J}-\frac{\partial U_{c e l l}}{\partial \delta l_{I J}} \widehat{\mathbf{u}}_{I J}$

where $\widehat{\mathbf{u}}_{I J}=\left(\mathbf{r}_{I}-\mathbf{r}_{J}\right) /\left\|\mathbf{r}_{I}-\mathbf{r}_{J}\right\|$ is the displacement vector connecting solid particles $I$ and $J$. The term $-\frac{\partial U_{c e l l}}{\partial \delta l_{I J}}$ is defined in the following manner [26]:

$-\frac{\partial U_{\text {cell }}}{\partial \delta l_{I J}}=\left\{\begin{array}{ll}-k_{L} \delta l_{I J}-\frac{T}{2}\left(\sum_{J=1}^{8} \delta l_{I J}+\sum_{M=1}^{8} \delta l_{J M}\right) & J \epsilon 1,2,3,4 \\ -k_{D} \delta l_{I J}-\frac{T}{2}\left(\sum_{J=1}^{8} \delta l_{I J}+\sum_{M=1}^{8} \delta l_{J M}\right) & J \in 5,6,7,8\end{array}\right.$, 
where $\delta l_{I J}$ is the change of length of the half spring. In order to couple the SPH and LSM methods, the solid particles in the vicinity of the solid-fluid interface are treated as dummy particles in the SPH solver. Depending on the wall boundary conditions, the velocities of these dummy particles can be calculated accordingly [29].

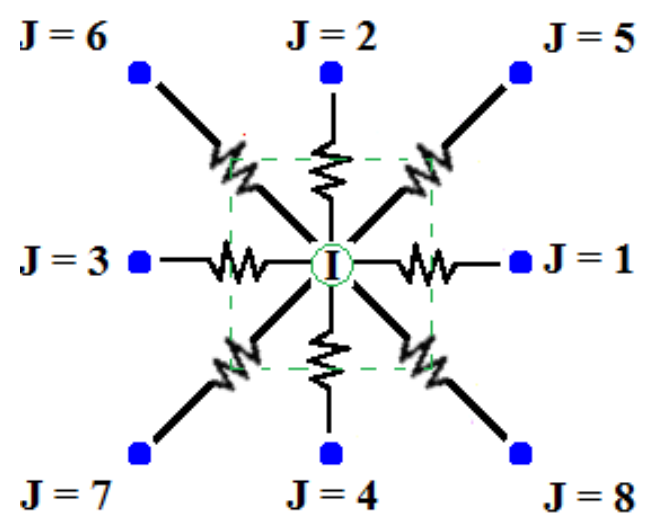

Fig. 1. Spring network in the solid body

\section{Results and Discussion}

The SPH-LSM solver has been developed and it was applied to solve the dam break flow involving a flexible structure. This test case is attractive as the experimental data of the gate displacement is available [30]. Also, previous researchers [31] have done validations on their SPH-FEM FSI solver using this test case as well. Figure 2 shows the size of the water column before collapsing, whereby its width $(W)$ and height $(H)$ were fixed at $0.1 \mathrm{~m}$ and $0.14 \mathrm{~m}$, respectively. The following fluid properties were employed: $\mu=0.001$ Pa.s and $\rho=1000 \mathrm{kgm}^{-3}$. There is a hinge at the top of the flexible gate. In order to model the hinge, the top elastic gate particles were connected with the neighbouring stationary dummy particles as shown in Figure 2. The thickness and the height of the flexible gate were fixed at $0.005 \mathrm{~m}$ and $0.079 \mathrm{~m}$, respectively. The material properties of the flexible gate were fixed as: $E=12 \mathrm{MPa}, v=0.40$ and $\rho=1100 \mathrm{kgm}^{-3}$. A uniform particle spacing of $1.0 \mathrm{~mm}$ was used. A monitor point was placed at the free end of the flexible gate. Its displacement with respect to time was then monitored as time progresses. The flow computation was executed until $t=0.4 \mathrm{~s}$.

Figure 3 compares the simulated displacement values against those obtained from the SPH-FEM solution [31]. Overall, the agreement is very encouraging. While the agreement between SPH-LSM and experimental data is good at $t<0.08 \mathrm{~s}$, our numerical results are somewhat lower than those measured at $t>0.08 \mathrm{~s}$. This could be due to the 2D plane-strain assumption made in our current model. In addition, we anticipate that the model can be further improved if the flexible gate is modelled using the hyperelasticity model, as Yang and his co-workers [31] have shown that the hyperelasticity model could give better accuracy. 


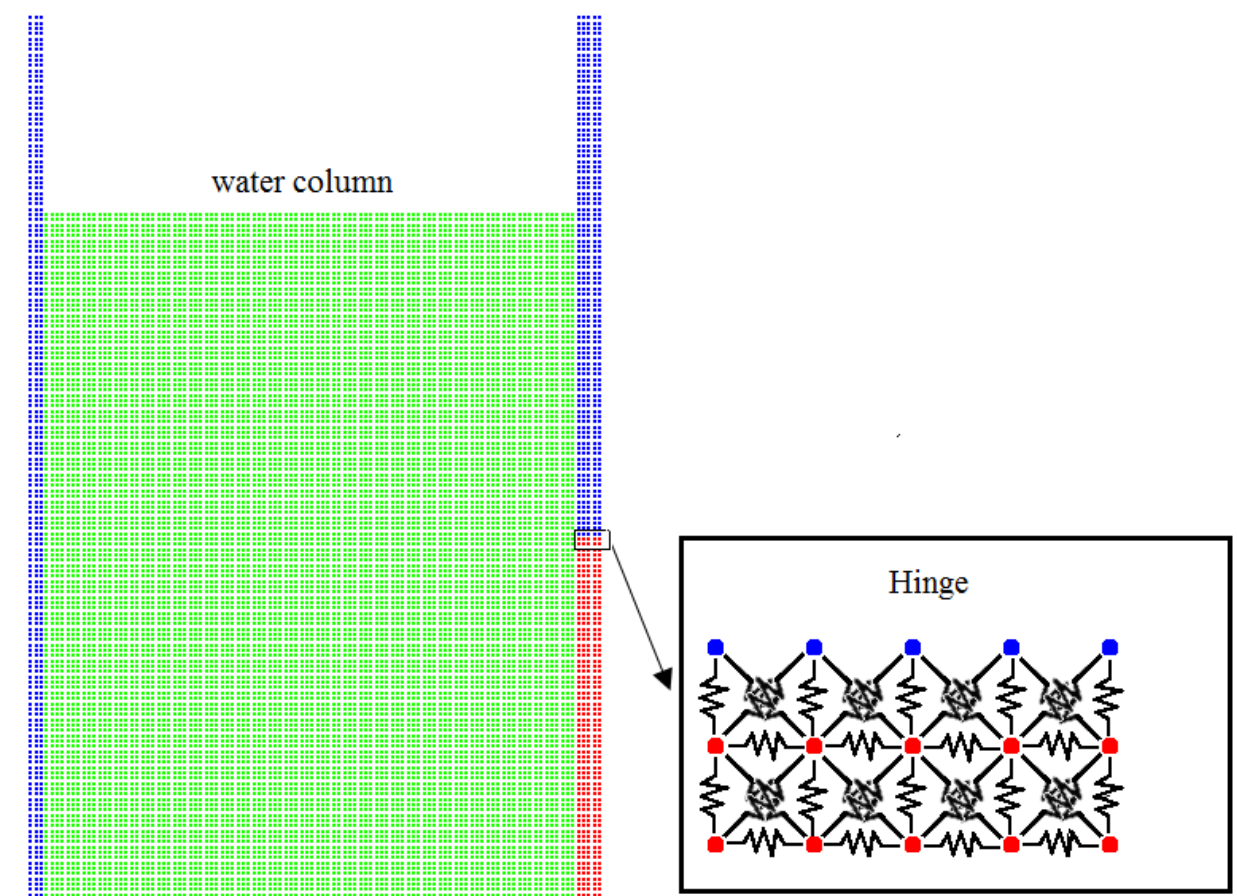

flexible gate

H:

Fig. 2. Initial positions of particles (red: flexible gate; blue: fixed wall; green: water)

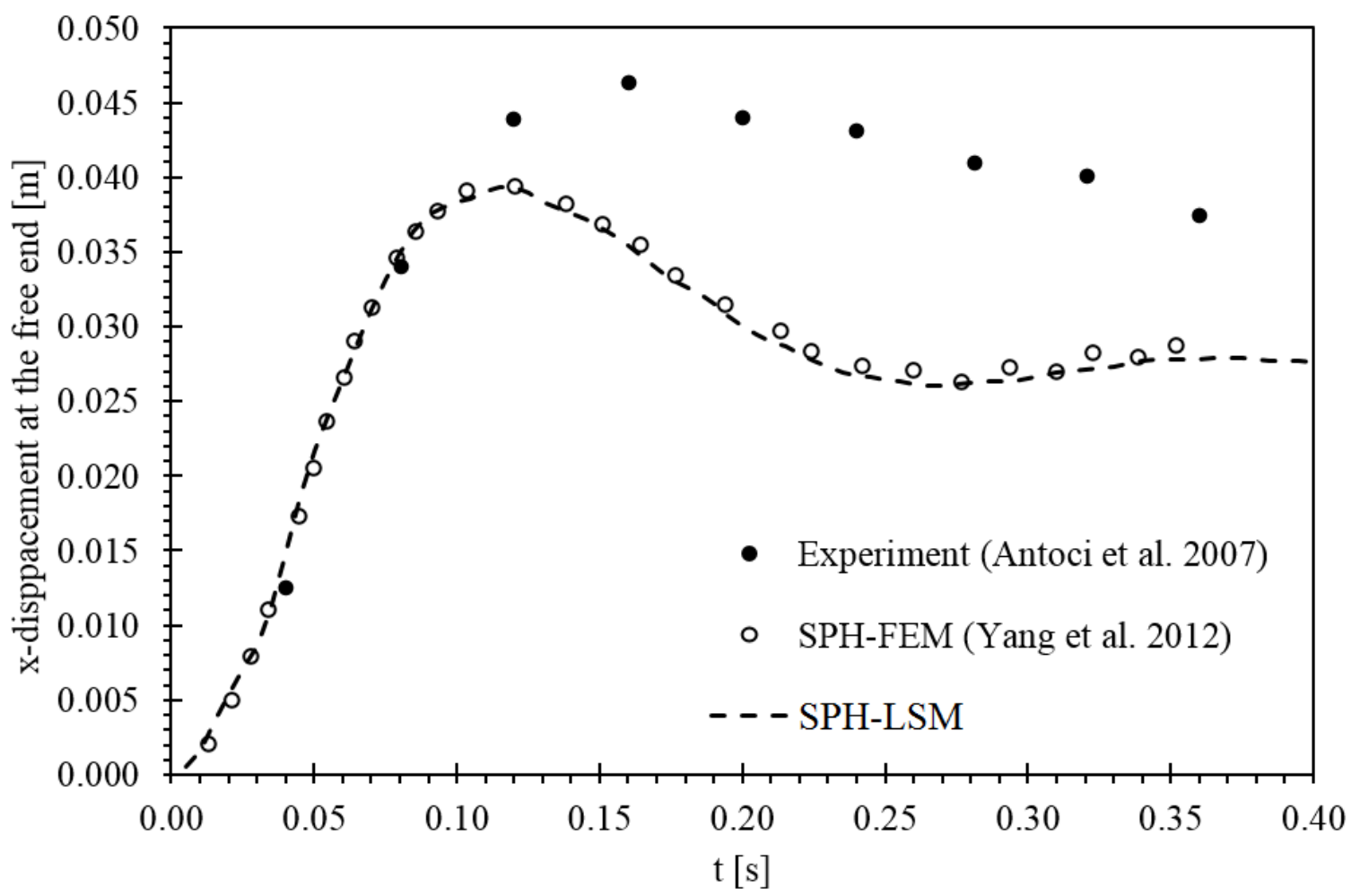

(a) 


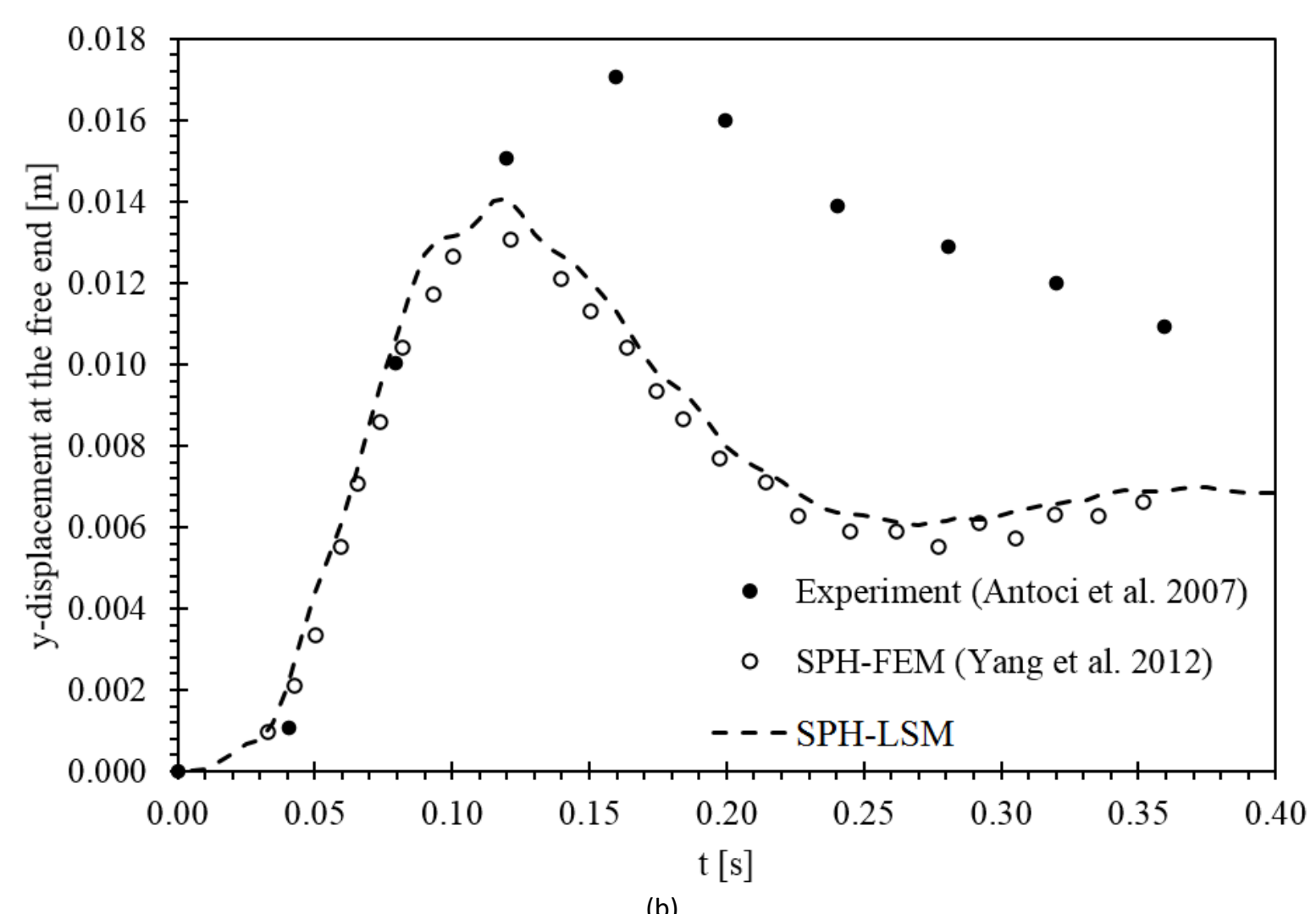

Fig. 3. $x-(a)$ and $y$ - (b) displacements at the free end of the flexible gate

\section{Conclusions}

The Smoothed Particle Hydrodynamics (SPH) method has been coupled with the Lattice Spring Model (LSM) method to model Fluid Structural Interaction (FSI) problem. The coupled method has been used to simulate dam break flow through a flexible gate. The numerical results have been compared against those obtained from the SPH-Finite Element Method (SPH-FEM) and good agreement has been found.

\section{Acknowledgement}

The authors would like to thank Takasako i-Kohza research fund [Vote No: R.K130000.7343.4B365] and Universiti Tenaga Nasional (UNITEN) for providing the computational facility in performing the simulation work.

\section{References}

[1] Tey, Wah Yen, Ryan Yuen Wye Hong, Yutaka Asako, Hooi Siang Kang, and Khai Ching Ng. "Analysis on Computational Efficiency of Convection Discretisation Schemes in SIMPLE Algorithm." Journal of Advanced Research in Fluid Mechanics and Thermal Sciences 58, no. 1 (2019): 100-117.

[2] Eymard, Robert, Thierry Gallouët, and Raphaèle Herbin. "Finite volume methods." Handbook of Numerical Analysis 7 (2000): 713-1018.

https://doi.org/10.1016/S1570-8659(00)07005-8

[3] Ng, Khai Ching, E. Y. K. Ng, M. Z. Yusoff, and T. K. Lim. "Applications of high-resolution schemes based on normalized variable formulation for 3D indoor airflow simulations." International Journal for Numerical Methods in Engineering 73, no. 7 (2008): 948-981.

https://doi.org/10.1002/nme.2106

[4] Barth, Timothy, Raphaèle Herbin, and Mario Ohlberger. "Finite volume methods: foundation and analysis." Encyclopedia of Computational Mechanics Second Edition (2018): 1-60. 
https://doi.org/10.1002/9781119176817.ecm2010

[5] Mukhtar, A., Khai Ching Ng, and Mohd Zamri Yusoff. "Design optimization for ventilation shafts of naturallyventilated underground shelters for improvement of ventilation rate and thermal comfort." Renewable Energy 115 (2018): 183-198. https://doi.org/10.1016/i.renene.2017.08.051

[6] Droniou, Jerome. "Finite volume schemes for diffusion equations: introduction to and review of modern methods." Mathematical Models and Methods in Applied Sciences 24, no. 08 (2014): 1575-1619. https://doi.org/10.1142/S0218202514400041

[7] Ng, Khai Ching. "A collocated finite volume embedding method for simulation of flow past stationary and moving body." Computers \& Fluids 38, no. 2 (2009): 347-357.

https://doi.org/10.1016/i.compfluid.2008.04.009

[8] Greenshields, C. J., and H. G. Weller. "A unified formulation for continuum mechanics applied to fluid-structure interaction in flexible tubes." International Journal for Numerical Methods in Engineering 64, no. 12 (2005): 15751593.

https://doi.org/10.1002/nme.1409

[9] Mukhtar, A., K. C. Ng, and M. Z. Yusoff. "Passive thermal performance prediction and multi-objective optimization of naturally-ventilated underground shelter in Malaysia." Renewable Energy 123 (2018): 342-352.

https://doi.org/10.1016/i.renene.2018.02.022

[10] Eymard, Robert, Tierry Gallouët, and Raphaele Herbin. "A cell-centred finite-volume approximation for anisotropic diffusion operators on unstructured meshes in any space dimension." IMA Journal of Numerical Analysis 26, no. 2 (2006): 326-353.

https://doi.org/10.1093/imanum/dri036

[11] Ng, Khai Ching, Mohd Zamri Yusoff, and Eddie Yin Kwee Ng. "Multigrid solution of Euler equations using highresolution NVD differencing scheme for unstructured meshes." Progress in Computational Fluid Dynamics, an International Journal 6, no. 7 (2006): 389-401. https://doi.org/10.1504/PCFD.2006.010964

[12] Ng, K. C., MA Abdul Aziz, and E. Y. K. Ng. "On the effect of turbulent intensity towards the accuracy of the zeroequation turbulence model for indoor airflow application." Building and Environment 46, no. 1 (2011): 82-88. https://doi.org/10.1016/j.buildenv.2010.07.002

[13] Xiang, Hao, and Bin Chen. "Simulating non-Newtonian flows with the moving particle semi-implicit method with an SPH kernel." Fluid Dynamics Research 47, no. 1 (2015): 1-27. https://doi.org/10.1088/0169-5983/47/1/015511

[14] Koshizuka, Seiichi, and Yoshiaki Oka. "Moving-particle semi-implicit method for fragmentation of incompressible fluid." Nuclear Science and Engineering 123, no. 3 (1996): 421-434. https://doi.org/10.13182/NSE96-A24205

[15] Ng, K. C., E. Y. K. Ng, and W. H. Lam. "Lagrangian simulation of steady and unsteady laminar mixing by plate impeller in a cylindrical vessel." Industrial \& Engineering Chemistry Research 52, no. 29 (2013): 10004-10014. https://doi.org/10.1021/ie400621b

[16] Liu, Kuan-Shuo, Tony Wen-Hann Sheu, Yao-Hsin Hwang, and Khai-Ching Ng. "High-order particle method for solving incompressible Navier-Stokes equations within a mixed Lagrangian-Eulerian framework." Computer Methods in Applied Mechanics and Engineering 325 (2017): 77-101. https://doi.org/10.1016/i.cma.2017.07.001

[17] Ng, K. C., T. W. H. Sheu, and Y. H. Hwang. "Unstructured Moving Particle Pressure Mesh (UMPPM) method for incompressible isothermal and non-isothermal flow computation." Computer Methods in Applied Mechanics and Engineering 305 (2016): 703-738. https://doi.org/10.1016/j.cma.2016.03.015

[18] Ng, K. C., Y. H. Hwang, Tony WH Sheu, and C. H. Yu. "Moving Particle Level-Set (MPLS) method for incompressible multiphase flow computation." Computer Physics Communications 196 (2015): 317-334. https://doi.org/10.1016/j.cpc.2015.06.021

[19] Ibrahim, Abdalla Mohamed Elfatih, Khai Ching Ng, and Yee Luon Ng. "On the Parallelization of Moving Particle Level Set (MPLS) Method for Multiphase Flow Simulation using OpenMP." CFD Letters 11, no. 1 (2019): 69-85.

[20] Groot, Robert D., and Patrick B. Warren. "Dissipative particle dynamics: Bridging the gap between atomistic and mesoscopic simulation." The Journal of Chemical Physics 107, no. 11 (1997): 4423-4435. https://doi.org/10.1063/1.474784

[21] Ng, Khai Ching, and T. W. H. Sheu. "Refined energy-conserving dissipative particle dynamics model with temperature-dependent properties and its application in solidification problem." Physical Review E 96, no. 4 (2017): 043302. 
https://doi.org/10.1103/PhysRevE.96.043302

[22] Monaghan, Joe J. "Smoothed particle hydrodynamics." Annual Review of Astronomy and Astrophysics 30, no. 1 (1992): 543-574. https://doi.org/10.1146/annurev.aa.30.090192.002551

[23] Ng, Khai Ching, Yee Luon Ng, T. W. H. Sheu, and A. Mukhtar. "Fluid-solid conjugate heat transfer modelling using weakly compressible smoothed particle hydrodynamics." International Journal of Mechanical Sciences 151 (2019): 772-784.

https://doi.org/10.1016/j.ijmecsci.2018.12.028

[24] Ng, K. C., Y. L. Ng, T. W. H. Sheu, and A. Alexiadis. "Assessment of Smoothed Particle Hydrodynamics (SPH) models for predicting wall heat transfer rate at complex boundary." Engineering Analysis with Boundary Elements 111 (2020): 195-205.

https://doi.org/10.1016/i.enganabound.2019.10.017

[25] Xia, Muming, Hui Zhou, Hanming Chen, Qingchen Zhang, and Qingqing Li. "A rectangular-grid lattice spring model for modeling elastic waves in Poisson's solids." Geophysics 83, no. 2 (2018): T69-T86. https://doi.org/10.1190/geo2016-0414.1

[26] Chen, Hailong, Enqiang Lin, and Yongming Liu. "A novel Volume-Compensated Particle method for 2D elasticity and plasticity analysis." International Journal of Solids and Structures 51, no. 9 (2014): 1819-1833.

https://doi.org/10.1016/i.ijsolstr.2014.01.025

[27] Chen, Hailong, Enqiang Lin, Yang Jiao, and Yongming Liu. "A generalized 2D non-local lattice spring model for fracture simulation." Computational Mechanics 54, no. 6 (2014): 1541-1558. https://doi.org/10.1007/s00466-014-1075-4

[28] Schoenberg, Isaac Jacob. "Contributions to the problem of approximation of equidistant data by analytic functions. Part B. On the problem of osculatory interpolation. A second class of analytic approximation formulae." Quarterly of Applied Mathematics 4, no. 2 (1946): 112-141. https://doi.org/10.1090/qam/16705

[29] Adami, Stefan, Xiangyu Y. Hu, and Nikolaus A. Adams. "A generalized wall boundary condition for smoothed particle hydrodynamics." Journal of Computational Physics 231, no. 21 (2012): 7057-7075. https://doi.org/10.1016/i.jcp.2012.05.005

[30] Antoci, Carla, Mario Gallati, and Stefano Sibilla. "Numerical simulation of fluid-structure interaction by SPH." Computers \& Structures 85, no. 11-14 (2007): 879-890. https://doi.org/10.1016/j.compstruc.2007.01.002

[31] Yang, Qing, Van Jones, and Leigh McCue. "Free-surface flow interactions with deformable structures using an SPHFEM model." Ocean Engineering 55 (2012): 136-147. https://doi.org/10.1016/j.oceaneng.2012.06.031 